\title{
Variabilité de la composition organique des huiles essentielles extraites d'organes de Monodora tenuifolia provenant de trois sites de récolte en Côte d'Ivoire
}

\author{
Manuscrit reçu le 26 août 2015 et acccepté le 29 mars 2016 \\ DJE BI Marc Gabin, KABRAN Guy Roger Mida, OUATTARA Zana Adama, KADJA Amani Brice, \\ MAMYRBEKOVA-BEKRO Janat Akhanovna ${ }^{1}$, BEKRO Yves-Alain
}

Laboratoire de Chimie Bio Organique et de Substances Naturelles, UFR-SFA, Université Nangui Abrogoua, 02 BP 801 Abidjan 02-République de Côte d'Ivoire

\section{Résumé}

Des échantillons d'huiles essentielles (HE) de feuilles, d'écorces de tronc et de racines de Monodora Tenuifolia (Benth.) (Annonaceae) provenant de trois sites de récolte en Côte d'Ivoire, ont été analysés par GC-MS. Les rendements et la composition chimique des HE obtenues par hydrodistillation varient en fonction des organes de la plante d'étude et des sites de récolte (I), (II), (III) respectivement Adiopodoumé, AnyamaAdjamé et Kongofon. Les organes de $M$. tenuifolia provenant du site (I) sont plus riches en HE que ceux des sites (II) et (III). Les rendements en $\operatorname{HE~}(0,47 \%, 0,32 \%, 0,36 \%)$ obtenus à partir des racines récoltées sur les sites (I), (II) et (III), sont plus élevés que ceux en HE des écorces de tronc $(0,06 \%, 0,08 \%, 0,05 \%)$ et de feuilles $(0,2 \%, 0,12 \%, 0,08 \%)$. Le nombre des composés identifiés dans les HE d'écorces de tronc (45, 46, 38 ) est supérieur à celui de ceux retrouvés dans les HE de racines $(40,40,40)$ et de feuilles $(27,38,28)$. La composition chimique est dominée par les sesquiterpènes et elle diffère selon les sites de récolte et les organes des plantes.

Mots clés : Monodora tenuifolia, huile essentielle, variabilité, GC-MS, Côte d'Ivoire

\begin{abstract}
Samples of essential oils (EO) of leaves, trunk and roots barks of Monodora Tenuifolia (Benth.) (Annonaceae) coming from three sites of harvest in Côte d'Ivoire, have been analyzed by GC-MS. The outputs and the chemical composition of the EO obtained by hydrodistillation vary according to the organ of the plant studied and to the sites of harvest (I), (II), (III) respectively Adiopodoume, Anyama-Adjame and Kongofon. $M$. tenuifolia organs coming from the site (I) are richer in EO that those from the sites (II) and (III). The outputs in EO $(0.47 \%, 0.32 \%, 0.36 \%)$ obtained from the roots harvested on the sites (I), (II) and (III), are higher than those in EO of the trunk barks $(0.06 \%, 0.08 \%, 0.05 \%)$ and of the leaves $(0.2 \%, 0.12 \% ; 0.08 \%)$. The number of the compounds identified in the EO of trunk barks $(45,46,38)$ is superior to the one of those recovered in the EO of roots $(40,40,40)$ and of leaves $(27,38,28)$. The chemical composition is dominated by the sesquiterpenes and it differs according to the sites of harvest and the organ of the plant.
\end{abstract}

Key words: Monodora tenuifolia, essential oil, variability, GC-MS, Côte d'Ivoire

${ }^{1}$ Adresses pour le courrier électronique : kojanova1926@hotmail.fr / amyrbekova.janat@lablcbosn.com 


\section{Introduction}

La flore africaine regorge d'un grand nombre de plantes aromatiques dont les HE font l'objet de nombreuses études [1-3]. Toutefois, les informations inhérentes à la variabilité de la composition chimique des HE extraites à partir des différents organes des plantes provenant de différents sites de récolte sont insuffisantes.

Monodora tenuifolia (Benth.) est une plante aromatique de la famille des Annonanceae des régions forestières des zones tropicales. Elle se repend en Inde, aux Antilles, en Malaisie et en Afrique [4]. En Afrique, elle est largement distribuée le long des côtes ouest [5]. En Côte d'Ivoire, cette espèce végétale est rencontrée dans toute la zone forestière et surtout dans les formations secondaires en voie de reforestation [6]. En médecine traditionnelle, les racines de $M$. tenuifolia sont employées pour traiter la dysenterie et les maux de dent [7-9]. Les graines rôties et broyées de $M$. tenuifolia sont frottées sur la peau pour soigner les affections cutanées [8]. En Côte d'Ivoire, M. tenuifolia est utilisé en association avec Monodora myristica pour la préparation d'un collyre contre diverses ophtalmies et troubles de la vision [10]. Les graines de M. tenuifolia sont utilisées comme épices pour relever la saveur des mets [11]. Dans certains pays d'Afrique Centrale (Cameroun, Gabon, République Démocratique du Congo) et de l'Ouest (Guinée, Nigeria), M. tenuifolia s'emploie comme plante ornementale [5,12]. Deux alcaloïdes ont été isolés des extraits de M. tenuifolia : un de type indolique [13,5], l'autre de type laurelliptine [14,15]. Des terpènes contenus dans les racines, les fruits et des acides gras extraits des graines de $M$. tenuifolia [11,16] ont été caractérisés [17]. La composition chimique des HE des feuilles de M. tenuifolia du Bénin [2] et celle des écorces de racines de l'espèce nigériane [9], ont été mises en évidence par GC-MS. Néanmoins, les HE des écorces de tronc de M. tenuifolia n'ont pas encore fait l'objet étude.

Au regard de l'insuffisance d'informations sur la variabilité de la composition organique des HE issues de différents organes de plantes et ce, en fonction des sites géographiques de récolte, l'objectif principal de la présente étude a été de faire une étude comparative des constituants organiques des HE obtenues par hydrodistillation à partir des feuilles, des écorces de tronc et des racines de $M$. Tenuifolia provenant de trois sites de récolte: Adiopodoumé, Anyama-Adjamé et Kongofon.

\section{Matériel et Méthodes}

\subsection{Matériel végétal et extraction des HE}

Les localités du Sud de la Côte d'Ivoire à savoir Adiopodoumé et d'Anyama-Adjamé du District autonome d'Abidjan) et Kongofon de la Région de la Mé, ont été respectivement les sites géographiques de récolte (I), (II), (III) des feuilles fraîches, d'écorces de tronc et de racines de Monodora tenuifolia. Les récoltes de ces organes ont été faites en juin 2015, lesquels ont été ensuite identifiés au Centre National de Floristique de 
l'Université Félix Houphouët-Boigny (Abidjan/Cocody) conformément à l'herbier disponible (numéro de référence 12480). Les HE desdits organes ont été extraites par hydrodistillation à l'aide d'un appareil de type Clevenger. Dans un alambic contenant de l'eau, chaque de la plante est déposé sur une grille, disposée à $5 \mathrm{~cm}$ au-dessus de l'eau. Les HE sont obtenues pendant 3h $30 \mathrm{~min}$ de distillation, ensuite séchées sur $\mathrm{CuSO}_{4}$ anhydre, puis conservées dans un congélateur à $-6^{\circ} \mathrm{C}$. Les rendements sont calculés selon la formule suivante :

$$
R d t=\frac{m_{H E}}{m_{0}} \times 100
$$

où $m_{H E}$ est la masse (en g) de l'HE extraite et $m_{0}$ celle de l'organe frais (en g).

\subsection{Analyse de la composition chimique des HE par GC-SM}

Les échantillons des HE ont été analysés à l'aide d'un chromatographe de type SHIMADZU série QP 2010SE muni d'une colonne Zebron ZB-5MS (20 $\mathrm{m} \times 0,18 \mathrm{~mm} \times 0,18 \mu \mathrm{m})$. Le gaz vecteur utilisé est l'hélium avec un débit de $0,9 \mathrm{ml} / \mathrm{s}$. Le four a été réglé de $70{ }^{\circ} \mathrm{C}$ à $270{ }^{\circ} \mathrm{C}$ avec un gradient de $4{ }^{\circ} \mathrm{C} / \mathrm{min}$ et maintenue à $280{ }^{\circ} \mathrm{C}$ pendant $20 \mathrm{~min}$. Les températures de l'injecteur et du détecteur ont été fixées à $280^{\circ} \mathrm{C}$. L'injection a été réalisée en mode splitless. La fragmentation a été effectuée par impact électronique de $70 \mathrm{eV}$; multiplicateur d'électrons, $2.500 \mathrm{~V}$. La température de la source d'ionisation a été fixée à $230{ }^{\circ} \mathrm{C}$ et la vitesse d'acquisition a été de 10.000 u.m.a./s. Les spectres de masse ont été obtenus en mode de balayage en m/z gamme 33-450. L'identification des constituants organiques des HE a été possible par comparaison de leurs spectres GC-MS avec ceux des principaux composés de référence tirés de la littérature $[18,19]$.

\section{Résultats et Discussion}

\subsection{Rendements en HE}

La Figure 1 indique les différents rendements d'extraction des HE à partir de chaque organe de M. tenuifolia . 


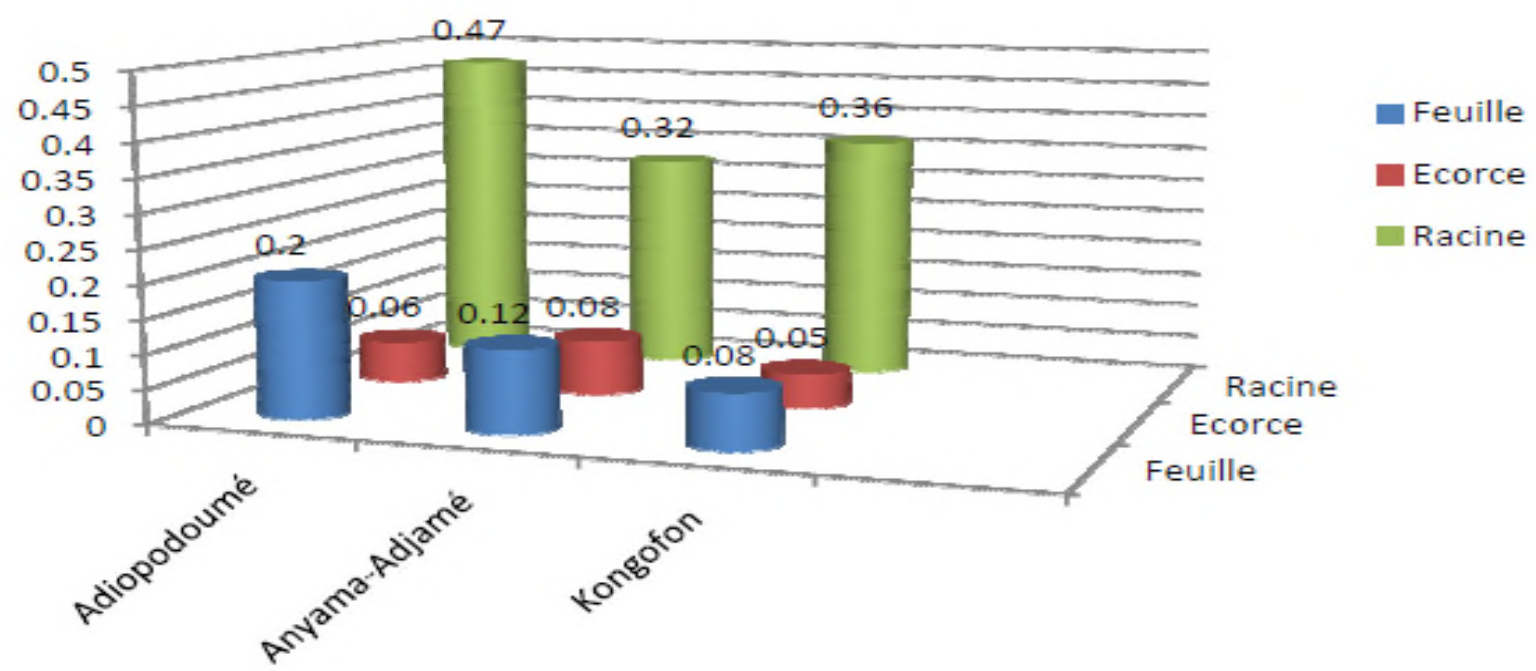

Figure 1: Rendements d'extraction des HE des feuilles, de tronc et de racines de Monodora tenuifolia.

Les HE des organes de Monodora tenuifolia obtenues par hydrodistillation et provenant des sites de récolte (I), (II) et (III) ont une couleur claire jaunâtre. Les rendements d'extraction en HE diffèrent en fonction des sites de récolte et des organes des plantes étudiées. En effet, les feuilles ont fourni 0,2\% (I), 0,12\% (II) et 0,08\% (III) d'HE. Les écorces de tronc et de racine en ont donné respectivement 0,06\% (I), 0,08\% (II), $0,05 \%$ (III) et 0,47\% (I), 0,32\% (II), 0,36\% (III). Ainsi, les rendements des HE provenant d'Adiopodoumé sont plus élevés que ceux d'Anyama-Adjamé et de Kongofon. Des résultats similaires ont été rapportés par d'autres auteurs qui ont montré l'influence du site de récolte sur le rendement en HE [20,21]. Aussi, la Figure 1 montre-t-elle bien que les écorces de racine (partie souterraine) produisent plus d'HE que les feuilles et les écorces de tronc (parties aériennes). Par ailleurs, en comparant les résultats que nous avons obtenus avec ceux d'autres auteurs, il ressort que quelque soit le lieu de récolte, les rendements en HE d'écorces de racines de Monodora tenuifolia de Côte d'Ivoire sont nettement supérieurs au rendement d'extraction des HE de l'espèce du Nigéria (0,03\%) [9]. Ce qui probablement s'expliquerait par les facteurs géographiques (zone de récolte, climat), le stade de croissance de la plante et les techniques d'extraction [22, 23].

\subsection{Composition organique des HE}

Les phytocomposés constituant la composition organique des HE des feuilles, d'écorces de tronc et de racines en fonction du site de récolte, sont regroupés dans le Tableau 1. 
Tableau 1: Composition organique des HE des différents organes de Monodora tenuifolia récoltés sur les sites (I), (II), (III).

\begin{tabular}{|c|c|c|c|c|c|c|c|c|c|c|c|}
\hline \multirow[t]{2}{*}{$n^{\circ}$} & \multirow[t]{2}{*}{ Phytocomposé identifié } & \multirow[b]{2}{*}{ [TR] } & \multicolumn{3}{|c|}{ Feuilles } & \multicolumn{3}{|c|}{ Écorces de tronc } & \multicolumn{3}{|c|}{ Écorces de racine } \\
\hline & & & Site I & Site II & Site III & Site I & Site II & Site III & Site I & Site II & Site III \\
\hline 1 & Hex-3-en-1-ol & 3,26 & & 0,19 & & & & & & & \\
\hline 2 & Hexylformiate & 3,41 & & & & & 0,16 & & & & \\
\hline 3 & $\alpha$-Thujène & 4,00 & & & & & 0,17 & & & & \\
\hline 4 & $1 \mathrm{R}-\alpha$-Pinène & 4,09 & 1,15 & 0,47 & 0,74 & 6,85 & 0,43 & 4,31 & 2,14 & 2,65 & 1,58 \\
\hline 5 & Camphène & 4,26 & & & 0,32 & 0,49 & & & 6,22 & 7,87 & 4,32 \\
\hline 6 & $\beta$-Pinène & 4,52 & & & 0,22 & 1,89 & & 1,30 & & & \\
\hline 7 & 6-Méthylhept-5-en-2-one & 4,56 & & & & & & 0,48 & & & \\
\hline 8 & $\beta$-Myrcène & 4,61 & & 1,15 & 1,09 & 1,09 & 0,97 & 0,34 & 0,31 & 0,58 & 0,29 \\
\hline 9 & $\delta$-Carène & 4,81 & & 0,92 & & 2,58 & 3,63 & 1,00 & & 0,20 & \\
\hline 10 & p-Cymène & 4,90 & & 0,26 & & & & & & & \\
\hline 11 & 4-éthyl-1,2-diméthylbenzène & 4,94 & & & & 1,17 & & & & & \\
\hline 12 & $\beta$-Cymène & 4,94 & & & & & & & & & 0,10 \\
\hline 13 & 4-Ethyl-o-xylène & 4,95 & & & & & & 0,66 & & & \\
\hline 14 & 1,2,3,4-Tétraméthylfulvène & 4,96 & & & & & 0,90 & & & & \\
\hline 15 & D-Limonène & 4,99 & & 0,91 & & 2,03 & 1,38 & 0,93 & 0,48 & 0,79 & 0,37 \\
\hline 16 & Non identifié & 5,02 & 1,85 & & & & & & & & 0,43 \\
\hline 17 & 7-Méthyloctan-4-ol acétate & 5,03 & & & 0,49 & & & & & & \\
\hline 18 & 1-Méthylheptylacétate & 5,05 & & & & 8,20 & & 2,28 & & & \\
\hline 19 & (E)- $\beta$-Ocimène & 5,06 & & 1,85 & & & & & 1,05 & 3,44 & \\
\hline 20 & 4-Carène & 5,09 & & 4,08 & & & 12,29 & & & & \\
\hline 21 & (Z)- $\beta$-Ocimène & 5,12 & & & & & & & 1,22 & 0,80 & 1,78 \\
\hline 22 & $\beta$-Ocimène & 5,13 & & & & 4,34 & 7,35 & 1,15 & & & \\
\hline 23 & $\gamma$-Terpinène & 5,23 & & 0,21 & & & 0,43 & & & 0,23 & 0,18 \\
\hline 24 & Octane-1-ol & 5,32 & & 0,23 & 0,23 & 0,67 & 0,96 & 1,23 & & & \\
\hline 25 & $\alpha$-Pinène époxyde & 5,34 & & & & 0,29 & & & & & \\
\hline 26 & (+)-4-Carène & 5,46 & & 0,63 & & & 1,50 & & & 0,12 & \\
\hline 27 & 1-Fenchone & 5,51 & & & & & & & & 0,09 & \\
\hline 28 & Linalol & 5,55 & 4,90 & 4,43 & 1,57 & 5,65 & 4,51 & 1,49 & 0,10 & 0,15 & \\
\hline 29 & (4E, 6Z)-Alloocimène & 5,78 & & 0,15 & & & 0,54 & & & 0,20 & \\
\hline 30 & (R)-(+)-Citronellal & 5,97 & & 4,05 & & & 0,89 & & & & \\
\hline 31 & Camphre & 5,98 & & & & & & & 0,23 & 2,19 & \\
\hline 32 & Longicyclène & 6,01 & & & & & & & 0,28 & & 0,34 \\
\hline 33 & Isopulegol & 6,06 & & 0,41 & & & & & & 0,16 & \\
\hline 34 & Butanoate d'hexyle & 6,24 & & & & & 0,30 & & & & \\
\hline 35 & Non identifié & 6,26 & & 1,28 & & & & & & & \\
\hline 36 & p-Cymen-8-ol & 6,29 & & & & 0,16 & & & & & \\
\hline 37 & Acétate d'octyle & 6,37 & & 0,68 & & & 0,73 & & & & \\
\hline 38 & Oct-1-yn-3-ol & 6,44 & & & & 0,32 & & & & & \\
\hline 39 & (R)- $\beta$-Citronellol & 6,50 & & 1,28 & & & 0,43 & & & 0,31 & \\
\hline
\end{tabular}




\begin{tabular}{|c|c|c|c|c|c|c|c|c|c|c|}
\hline 2-Méhylbutanoate d'hexyle & 6,55 & & & & 0,22 & & & & & \\
\hline Bergaptol & 6,65 & & & & 0,29 & 0,25 & & & & \\
\hline Acétate de bornyle & 6,94 & & 0,15 & & & & & & & \\
\hline Borneol & 6,99 & & & & 0,18 & 0,16 & & & & \\
\hline Hexylcyclobutane carboxylate & 7,18 & & & & 0,31 & & 0,98 & & & \\
\hline$\delta$-Elemène & 7,28 & 0,76 & & & & & & & & \\
\hline$\alpha$-Cubebène & 7,36 & & 0,70 & 0,31 & 1,45 & 0,23 & 0,62 & & & 0,12 \\
\hline Copaene & 7,57 & 2,11 & 2,92 & 1,76 & 7,09 & 0,80 & 5,55 & 0,52 & & 0,70 \\
\hline Isoledène & 7,60 & & & & 2,25 & & & & & \\
\hline Non identifié & 7,64 & & & 1,58 & & & & & & \\
\hline$\beta$-Cubebène & 7,67 & 2,12 & & & & & & & & \\
\hline$\beta$-Elemène & 7,73 & & 5,47 & 23,82 & & 2,71 & 4,42 & 2,21 & 1,33 & 3,56 \\
\hline$\beta$-Patchoulène & 7,75 & & 0,26 & 0,38 & & & & 8,50 & & 16,39 \\
\hline (-) $\alpha$-Gurjunène & 7,78 & & & & 3,06 & & & & & \\
\hline$\beta$-Bergamotène & 7,80 & 3,81 & & & & & & & & \\
\hline (-)-Cypérène & 7,83 & & & & & 9,69 & 15,87 & & 5,81 & \\
\hline$\alpha$-Santalène & 7,85 & 0,88 & & & 15,54 & & & 8,98 & 2,13 & \\
\hline $\begin{array}{l}\text { 1,7-Dimethyl-7-(4-methyl-3- } \\
\text { pentenyl)tricyclo }[2 \cdot 1 \cdot 0(2,6)] \text { heptane }\end{array}$ & 7,86 & 38,02 & & & & & & & & \\
\hline$\beta$-Caryophyllène & 7,87 & 8,11 & 6,75 & 10,66 & 2,20 & 3,64 & 6,15 & 1,14 & 0,63 & 2,25 \\
\hline$\beta$-Bergamotène & 7,88 & & & & 1,24 & & & 0,91 & & \\
\hline Non identifié & 7,92 & & 0,71 & 0,83 & & & & & & \\
\hline Non identifié & 7,94 & & & & 0,47 & & 0,92 & & & \\
\hline$\beta$-Farnesène & 7,98 & & & & & & & 1,85 & 1,65 & 1,34 \\
\hline$\beta$-Bisabolène & 7,99 & 4,50 & 1,08 & & 1,65 & & & & & \\
\hline $\begin{array}{l}\text { 2-méthyl-3-méthylène-2(4-méthyl-3- } \\
\text { pentényl) bicyclo [2.2.1]heptane }\end{array}$ & 8,00 & 3,19 & & & & & & & & \\
\hline Aromadendrene & 8,01 & & & 0,78 & & & & & & \\
\hline Non identifié & 8,02 & & & & & & & 2,25 & 1,29 & 2,10 \\
\hline$\alpha$-Gurjunène & 8,03 & & & & & 0,56 & 1,01 & & & \\
\hline$\gamma$-Muurolène & 8,05 & & & 0,41 & & & & & & \\
\hline$\beta$-Santalène & 8,06 & 1,92 & & & 1,31 & & & & & \\
\hline$\alpha$-Caryophyllène & 8,08 & & 1,09 & & & & & 1,44 & & 1,02 \\
\hline $\begin{array}{l}\text { (Z,Z,Z)-1,5,9,9-Tétraméthyl } \\
\text { cycloundeca-1,4,7-triène }\end{array}$ & 8,09 & 1,86 & & 2,96 & 2,09 & & 1,13 & & 0,96 & \\
\hline $\begin{array}{l}\text { 1,7,7-triméthylbicyclo[2,2,1]hept-5- } \\
\text { én-2-ol }\end{array}$ & 8,10 & & & & & 1,30 & 2,50 & & & 2,87 \\
\hline $\begin{array}{l}\text { 2-Butènedioate de bis[(2-methyl-1- } \\
\text { cyclohexen-1-yl)methyle] }\end{array}$ & 8,13 & & & & & & & 1,92 & 0,87 & \\
\hline Non identifié & 8,16 & 1,17 & & & 2,94 & & & & & \\
\hline Valencène & 8,17 & & & & & & 3,97 & & & \\
\hline$\alpha$-Guaiène & 8,19 & & & & 0,54 & & & 14,83 & 12,03 & 15,09 \\
\hline Germacrène D & 8,22 & 2,93 & & & & & & & & \\
\hline cyclodeca-1,6-diène & 8,24 & & & & 1,98 & & & & & \\
\hline$\alpha$-Farnesène & 8,27 & 5,92 & & & 1,22 & & 3,10 & & & \\
\hline$\beta$-Selinène & 8,29 & & & & & & & 1,66 & & 2,03 \\
\hline
\end{tabular}


Bulletin de la Société Royale des Sciences de Liège, Vol. 85, 2016, p. 17 - 29

\begin{tabular}{|c|c|c|c|c|c|c|c|c|c|c|c|}
\hline 82 & $\alpha$-Muurolène & 8,30 & & & & & & 2,12 & & & \\
\hline 83 & $\beta$-Cubebène & 8,31 & & 39,07 & 45,62 & & 6,57 & 7,92 & & & \\
\hline 84 & $\alpha$-Selinène & 8,32 & & & & & & 1,95 & & & \\
\hline 85 & $\beta$-Humulène & 8,32 & & & & & 3,21 & & & & \\
\hline 85 & $\gamma$-Bisabolène & 8,33 & 5,26 & & & & & & & & \\
\hline 87 & Non identifié & 8,33 & & & & 2,18 & & & & & \\
\hline 88 & $\alpha$-Bergamotène & 8,34 & & & & & & & 7,17 & 12,58 & 8,54 \\
\hline 89 & Elixène & 8,35 & & 2,87 & & & & & & & \\
\hline 90 & $\delta$-Cadinène & 8,41 & 1,31 & 2,99 & 2,67 & & & & 1,84 & & \\
\hline 91 & $\beta$-Cadinène & 8,42 & & & & 4,76 & 1,69 & 11,60 & & & \\
\hline 92 & Sesquiphellandrène & 8,43 & & & & & & & & 1,39 & \\
\hline 93 & Isoledène & 8,43 & & & & & & & & & 3,46 \\
\hline 94 & L-Calamenène & 8,45 & & & & 0,68 & & & & & \\
\hline 95 & $\gamma$-Himachalène & 8,46 & & & & & 0,89 & & & & \\
\hline 96 & $\alpha$-Panasène & 8,47 & & & & & & & & 1,50 & \\
\hline 97 & $\begin{array}{l}\text { 3,3,7,11-tétramethyltricyclo } \\
{[6.3 \cdot 0.0(2,4)] \text { undec-8-ène }}\end{array}$ & 8,47 & & & & & & & 2,27 & & 2,71 \\
\hline 97 & $\beta$-Guaiène & 8,48 & & & & & & 1,28 & & & \\
\hline 99 & $\alpha$-Longipinène & 8,50 & & & 0,26 & & & & & & \\
\hline 100 & $\beta$-Maalène & 8,51 & & & & & & & & & 0,28 \\
\hline 101 & $\gamma$-Muurolène & 8,52 & & 0,77 & & & & & & & \\
\hline 102 & 8,9-Dehydrocycloisolongifolène & 8,53 & & & & & & & 1,39 & & \\
\hline 103 & $\alpha$-Calacorène & 8,56 & & & & 0,51 & & 1,88 & & & \\
\hline 104 & $\begin{array}{l}\text { (E)- 3,7,11-triméthyldodeca-1,6,10- } \\
\text { triène-3-ol }\end{array}$ & 8,58 & & & & 0,61 & & & & & \\
\hline 105 & $\beta$-elemol & 8,58 & & & 0,26 & & & & & & 0,84 \\
\hline 106 & trans Nerolidol & 8,59 & 1,36 & & & & & & & & \\
\hline 107 & Epiglobunol & 8,59 & & & & & & & 0,96 & & \\
\hline 108 & Longifolenaldehyde & 8,59 & & & & & 2,67 & & & & \\
\hline 109 & Dehydroaromadendrène & 8,62 & & & & & & & 1,11 & 0,80 & 0,22 \\
\hline 110 & Cadala-1,3,8-triène & 8,67 & & & & & & 1,03 & & & \\
\hline 111 & Germacrène $\mathrm{B}$ & 8,69 & & 4,03 & 0,47 & & 2,08 & & & & \\
\hline 112 & Germacrène D-4-ol & 8,77 & & & 0,66 & & & & & & 0,25 \\
\hline 113 & Oxyde de caryophyllène & 8,82 & & 0,59 & 0,61 & 1,27 & 0,97 & 3,36 & & & 0,87 \\
\hline 114 & Viridiflorol & 8,95 & 1,17 & & & & & 0,71 & 0,61 & & \\
\hline 115 & $\begin{array}{l}\text { 2-(1,4,4-Triméthylcyclohex-2- } \\
\text { enyl)éthanol }\end{array}$ & 8,87 & & & & & & 0,57 & & & \\
\hline 116 & Cubenol & 8,97 & & & & & 0,34 & & & 0,70 & \\
\hline 117 & $\beta$-Spathulenol & 9,00 & & & & & 0,18 & & & & \\
\hline 118 & $\begin{array}{l}\text { 7R,8R-Hydroxy-4-isopropylidè-ne- } \\
\text { 7-méthylbicyclo[5.3.1]undec-1-ène }\end{array}$ & 9,02 & & & & & & & & 0,99 & 0,60 \\
\hline 119 & Spathulenol & 9,03 & 0,38 & & & 2,26 & & 1,03 & & 0,21 & 0,38 \\
\hline 120 & Diepi- $\alpha$-cedrène & 9,04 & & & & & 0,54 & & & & \\
\hline 121 & $\tau$-Muurolol & 9,13 & & 1,52 & 0,55 & & & & & & 7,79 \\
\hline 122 & $\begin{array}{l}\text { 6-Isopropenyl-4,8a-dimethyl- 1,2,3, } \\
5,6,7,8 \text { a-octahydronaphtalen-2-ol }\end{array}$ & 9,15 & & & & & 0,75 & & 6,61 & & \\
\hline 123 & Non identifié & 9,17 & 1,30 & & & & & & & & \\
\hline \multirow[t]{2}{*}{124} & $\alpha$-Cadinol & 9,19 & & 0,77 & 0,60 & & 0,60 & 1,06 & & & \\
\hline & & & & & 23 & & & & & & \\
\hline
\end{tabular}




\begin{tabular}{|c|c|c|c|c|c|c|c|c|c|c|c|}
\hline 125 & Selina-6-en-4-ol & 9,20 & & & & & & & & & 0,47 \\
\hline 126 & Murolan-3,9-diene-10-peroxyde & 9,22 & & & & & & 1,02 & & & \\
\hline 128 & $\begin{array}{l}\text { trans - 4-(1-Méthylethyl) } \\
\text { cyclohexaneméthanol }\end{array}$ & 9,27 & 1,96 & & & & & & & & \\
\hline 127 & Epi-globulol & 9,28 & & & & & & & 0,49 & 0,51 & 0,61 \\
\hline 129 & Widdrol & 9,28 & & & & 0,73 & & & & & \\
\hline 130 & $\begin{array}{l}\text { 4-(2,2-Dimethyl-6- } \\
\text { methylenecyclohexy)butanal }\end{array}$ & 9,29 & & & & & & & 1,16 & & \\
\hline 131 & $\alpha$-Bisabolol & 9,32 & 1,27 & & & 0,57 & & & 0,45 & 0,43 & \\
\hline 132 & (+)-Cadiène & 9,35 & & & & & & & 0,39 & & 0,59 \\
\hline 133 & 8,9-Dehydrocycloisolongifolene & 9,37 & & 2,57 & & & 1,50 & & & & \\
\hline 134 & $\begin{array}{l}\text { 7-Méthyl-4(1-méthylethylidene) } \\
\text { bicyclo[5.3.1]undec-1-en-8-ol }\end{array}$ & 9,39 & & & & & & 0,79 & & & \\
\hline 135 & $\begin{array}{l}\text { 1,7-Diméthyl-4 } \alpha \text {-isopropényl } \\
\text { bicyclo[4.4.0]dec-6-en-9 } \beta \text {-ol }\end{array}$ & 9,40 & & & & & & & & 1,09 & \\
\hline 136 & Farnesol & 9,42 & 0,41 & & & & & & & & \\
\hline 137 & $\begin{array}{l}\text { 3,5,6,7,8,8a-hexahydro-4-8a- } \\
\text { dimethyl-6-(1-methylethenyl) } \\
\text { 2(H)naphtalenone }\end{array}$ & 9,44 & & & & & & & 0,52 & 0,50 & \\
\hline 138 & $\begin{array}{l}\text { 2,5,9-Triméthylcycloundeca-4,8- } \\
\text { diénone }\end{array}$ & 9,47 & & & & 1,11 & & & & & \\
\hline 139 & Santanol & 9,51 & & & & & & & 6,44 & 11,95 & 6,12 \\
\hline 140 & 4-(3-Méthylbut-2-ényl)-1H-indole & 9,97 & & 1,26 & & 1,35 & 17,27 & 2,28 & 9,60 & 20,69 & 8,15 \\
\hline \multicolumn{2}{|c|}{ Monoterpènes hydrocarbonés } & & 1,15 & 10,63 & 2,37 & 19,27 & 28,69 & $\mathbf{9 , 0 3}$ & 11,92 & 16,88 & 8,62 \\
\hline \multicolumn{2}{|c|}{ Sesquiterpènes hydrocarbonés } & & 77,63 & 68,00 & 86,88 & 43,47 & 32,61 & 67,94 & 52,72 & 39,05 & 55,12 \\
\hline \multicolumn{2}{|c|}{ Monoterpènes oxygénés } & & 4,90 & 10,32 & 1,57 & 6,28 & 5,99 & 1,49 & $\mathbf{0 , 3 3}$ & 2,90 & $\mathbf{0 , 0 0}$ \\
\hline \multicolumn{2}{|c|}{ Sesquiterpènes oxygénés } & & 4,59 & 2,88 & 2,68 & 4,83 & 4,76 & 6,76 & 10,06 & 14,60 & 10,65 \\
\hline \multicolumn{2}{|c|}{ Dérivés indoliques } & & $\mathbf{0 , 0 0}$ & 1,26 & $\mathbf{0 , 0 0}$ & 1,35 & 17,27 & 2,28 & 9,60 & 20,69 & 8,15 \\
\hline \multicolumn{2}{|c|}{ Composés non isopréniques } & & & 1,10 & $\mathbf{0 , 7 2}$ & 12.87 & 2.15 & 4.97 & & & \\
\hline \multicolumn{2}{|c|}{ Autres composés } & & 11,33 & 4,61 & 5.53 & 8,61 & 8,44 & 7.52 & 14,62 & 5,70 & 16,15 \\
\hline \multicolumn{2}{|c|}{ Total } & & 99,60 & $\mathbf{9 8 , 8}$ & 99,75 & 96,68 & 99,91 & 99,99 & 99,25 & $\mathbf{9 9 , 8 2}$ & 98,69 \\
\hline
\end{tabular}

107 phytocomposés organiques ont été identifiés dans les HE de M. tenuifolia provenant d'Adiopodoumé, dont 27, 44 et 36 respectivement dans les HE de feuilles, des écorces de tronc et de racines. En ce qui concerne les organes issus d'Anyama-Adjamé, 116 composés ont été identifiés, dont 36, 42 et 38 respectivement dans les feuilles, les écorces de tronc et de racines. Quant au site de récolte de Kongofon, au nombre de de 101 composés trouvés, 26 sont issus des feuilles, 38 des écorces de tronc et 37 des écorces de racines. Les compositions organiques des HE obtenues font ressortir 6 groupes de phytocomposés : les monoterpènes et sesquiterpènes hydrocarbonés, les monoterpènes et sesquiterpènes oxygénés, les dérivés indoliques et d'autres composés (Figures 2-7). 


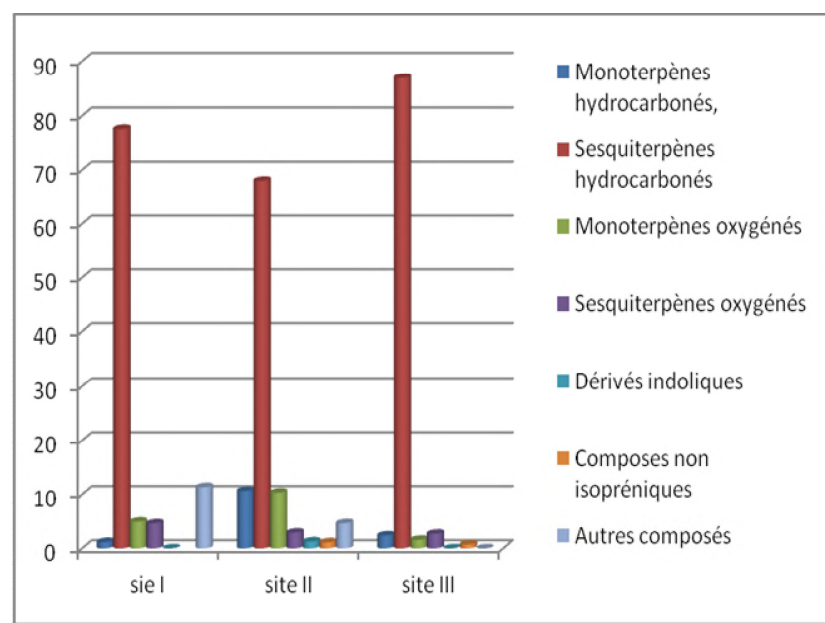

Figure 2: Composition organique des HE des feuilles de M. tenuifolia issues des sites de récolte (I), (II), (III)

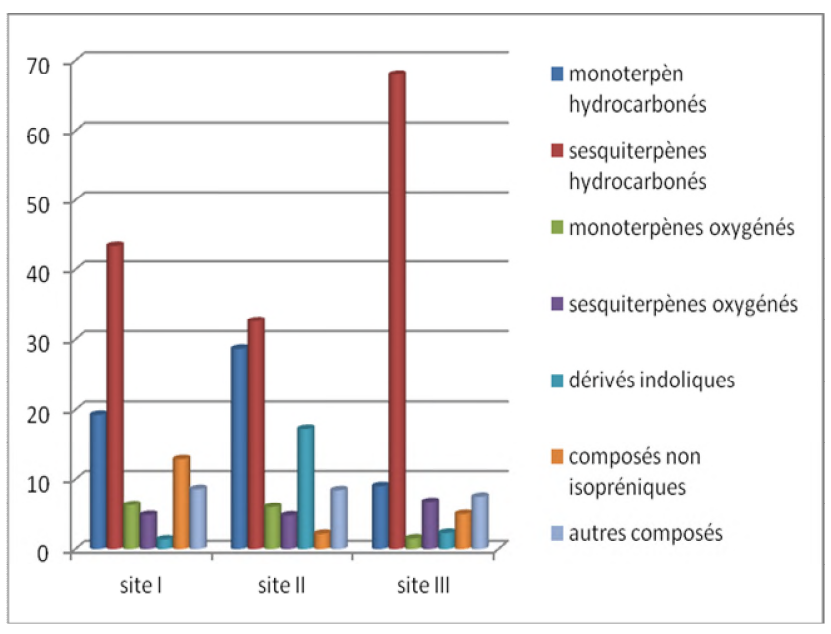

Figure 3: Composition organique des HE d'écorces de tronc de $M$. tenuifolia issues des sites de récolte (I), (II), (III)

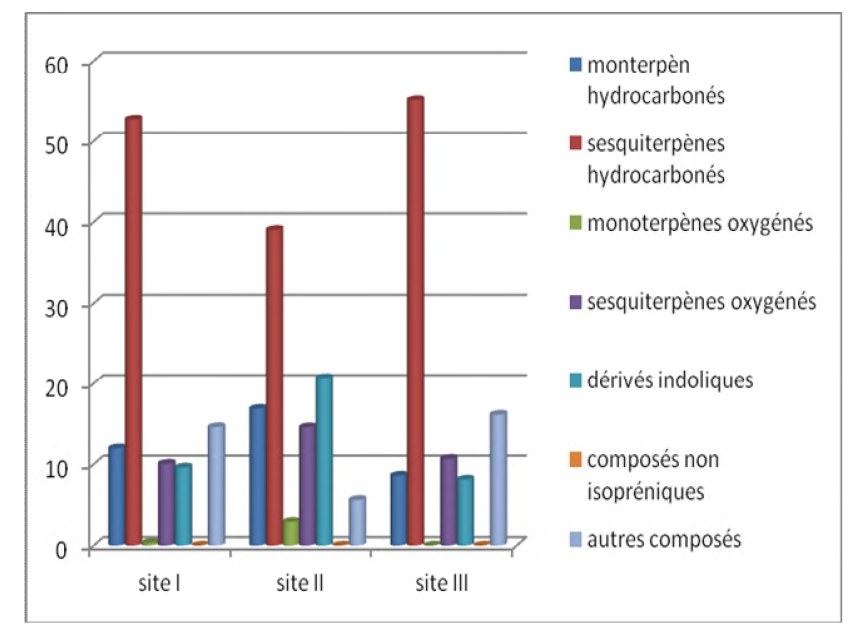

Figure 4: Composition organique des HE des écorces de racines de M. tenuifolia issues des sites de récolte (I), (II), (III)

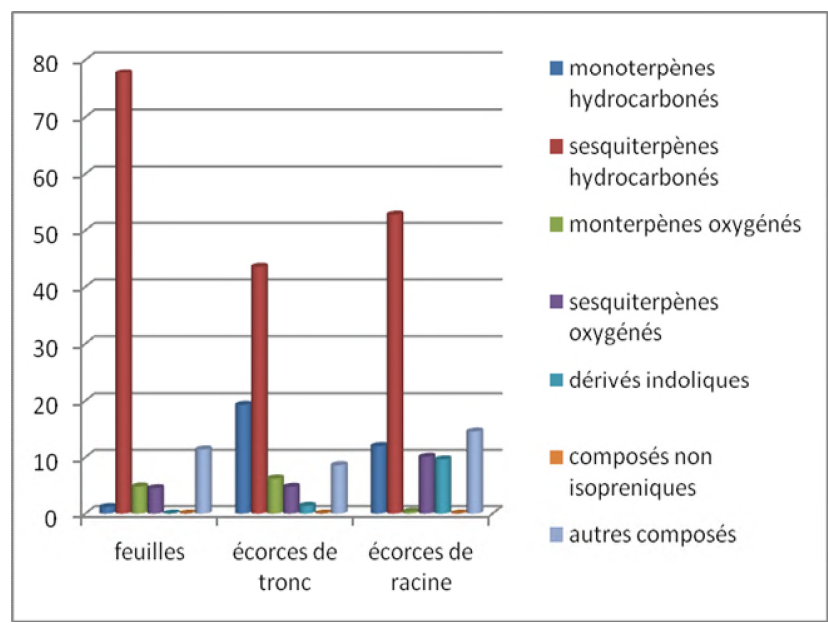

Figure 5: Composition organique des HE provenant du site de récolte (I)

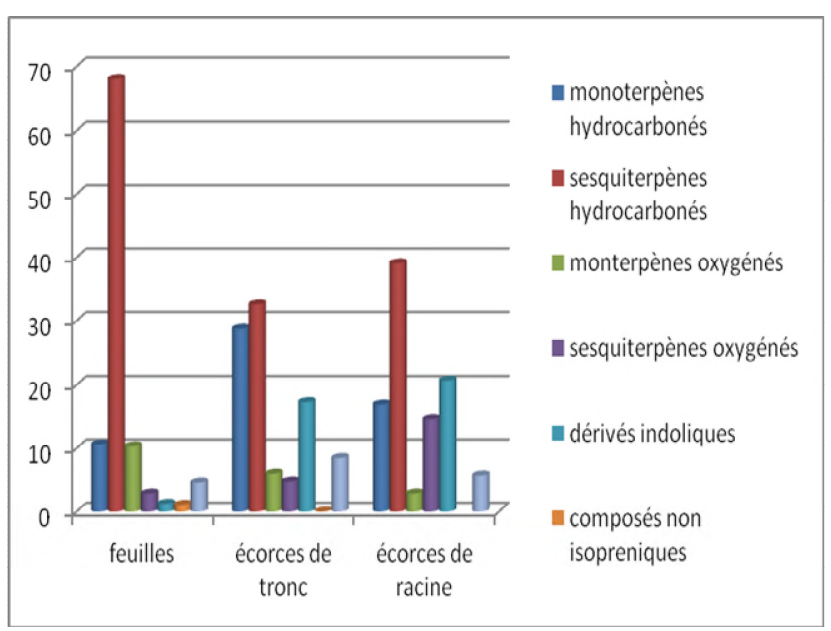

Figure 6: Composition organique des HE provenant du 25 site de récolte (II) 


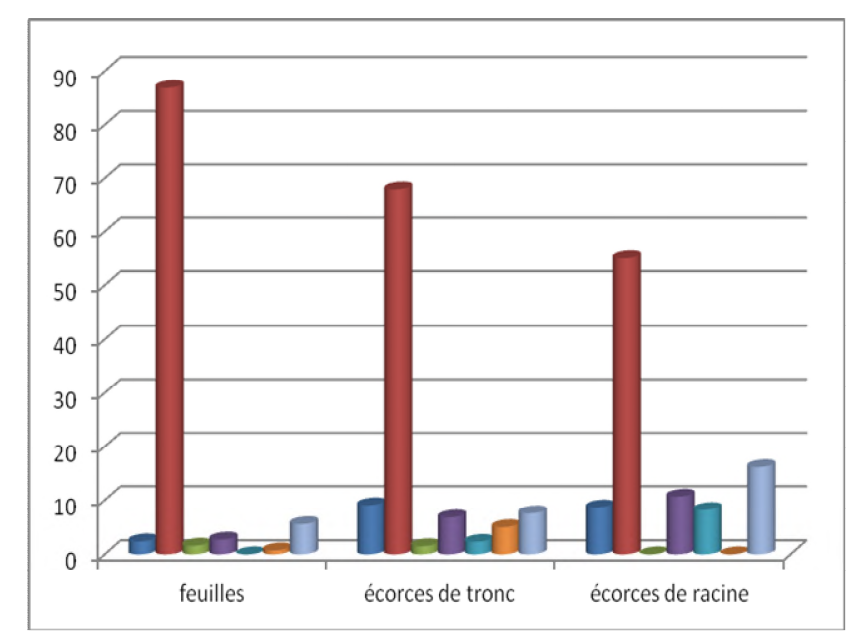

Figure 7: Composition organique des HE provenant du site de récolte (III)

Il est constaté au regard des Figures 2-7, que la composition organique des HE issues de différents organes de $M$. tenuifolia peut varier en fonction du site de collecte. De fait, les profils chimiques des HE de M. tenuifolia mettent nettement en évidence la variabilité organique desdites huiles quel que soit le site de récolte et l'organe de la plante. En général, une dominance des sesquiterpènes dans la composition organique de chaque HE est observée. Aussi remarquons-nous que les HE de la partie de la plante récoltée à AnyamaAdjamé (Figure 6) est plus riche en monoterpènes que celles provenant d'Adiopodoumé et de Kogofon (Figures 5 et 7). Concernant les 3 sites de récolte, la présence notable des monoterpènes dans les écorces de tronc et de racines est manifeste (Figure 6). Les HE des écorces de tronc et de racines provenant des 3 sites de récolte, contiennent des dérivés indoliques hormis celles extraites des feuilles. Ces phytocomposés sont plus abondants dans les HE des organes de M. tenuifolia provenant d'Anyama-Adjamé. Leur teneur 9,60 \% (I); 20,69 \% (II) et 8,15\% (III) est plus élevée dans les écorces de racines que dans les écorces de tronc 1,35\% (I); $17,27 \%$ (II) et 2,28\% (III). Ces résultats ne contredisent pas ceux d'auteurs nigérians [9] qui ont révélé la présence de composés indoliques $(15,3 \%)$ dans les écorces de racines.

Les composés majoritaires identifiés que renferment les HE des 3 organes de la plante étudiée, provenant des 3 sites de récolte sont regroupés dans le Tableau 1 à partir duquel nous remarquons que les composés majoritaires des HE du site (I) ne sont pas les mêmes que ceux des sites (II) et (III).

La présente étude montre par ailleurs, que le profil chimique des HE de l'espèce ivoirienne est manifestement différent de celui de l'espèce béninoise dont les composés majoritaires sont : $\alpha$-cadinol $(20,5 \%)$, germacrène D-4-ol (16,8 \%), muurolol (14,7\%), $\delta$-cadinène $(12,0 \%)$ et $\beta$-caryophyllène $(5,8 \%)$ [2]. 
L'analyse de la composition organique des HE des écorces de racines récoltées sur les 3 sites, montre une certaine homogénéité au niveau des composés majoritaires (Tableau 1). Toutefois, nos résultats s'éloignent complètement de ceux obtenus par d'autres chercheurs qui ont étudié l'espèce nigériane dont les composés majoritaires sont : camphène $(6,6 \%), E$ - $\beta$-ocimène $(2,8 \%), \alpha$-gurjunène $(2,2 \%), \beta$-selinène $(2,0 \%)$, nérolidol $(4,0 \%), \beta$-bisabolène $(2,4 \%)$ et 2 sesquiterpènes hydrogénés $(6,8 \%$ et $8,5 \%), 2$ sesquiterpènes oxygénés $(7,7 \%$ et $2,2 \%)$, dérivés indoliques $(15,3 \%)$ [9].

\section{Conclusion}

Monodora tenuifolia (Benth.) est une Annonaceae aromatique diversement utilisée en médecine traditionnelle. L'analyse par CPG-MS des HE des différents organes de plante issus des trois localités de récolte de Côte d'Ivoire a montré une variabilité dans les profils organiques tant au niveau qualitatif que quantitatif. Ces derniers sont dominés par les sesquiterpènes. Cependant, les monoterpènes sont aussi bien présents dans les HE de Monodora tenuifolia récolté à Anyama-Adjamé. Grosso modo, les profils organiques des HE des écorces de racines provenant des 3 sites de récolte, révèlent une certaine homogénéité au niveau des composés majoritaires. Afin d'établir le chémotype organique de Monodora tenuifolia, une étude plus large sur un grand nombre d'échantillons en fonction des saisons et des sites de récolte, est en cours de réalisation. Ce qui permettrait de démontrer une corrélation entre la composition organique et l'usage en médecine traditionnelle des différents organes de Monodora tenuifolia.

\section{Références}

1. Koumaglo K.H., Dotse K., Bettini F. et Bayle J.-C. Composition chimique de l'huile essentielle de Chromolaena odorata (L) King et Robinson (Asteraceae) du Togo : Effets de séchage et du site de récolte. J. Soc. Ouest-Afr. Chem., 28, 2009, 11-16

2. Tchobo F.P., Alitonou G.A., Soumanou M. M., Barea B., Bayrasy C., Laguerre M., Lecomte J. Villeneuve P., and Souhounhloue K.D, Chemical Composition and Ability of Essential Oils from six Aromatic Plants to counteract Lipid Oxidation in Emulsion, J. Am. Oil Chem. Soc., 91, 2014, 471-479.

3. Owokotomo I. A. and Ekundayo O.. Comparative study of the essential oils of Monodora myristica from Nigeria. Eur. Chem. Bull., 1, 2012, 263-265.

4. Talaji, S.J, Essential oil from Monodora myristica grown in Ghana, West A fr Pharm, 4, 1965, 64-65.

5. Adeoyé A.O., Oguntimein B.O., and Clark A.M., Hufford C.D., 3-dimethylallylindole: an anthibacterial and antifungal metabolite from Monodora tenuifolia, J. Nat Prod, 1986, vol 49, 534-537. 
6. Adjanohoun E. et Aké Assi L., Contribution au recensement des plantes médicinales de Côte d'Ivoire. Agence de Coopération Culturelle et Technique (ACCT) ; 1979, Tome I, 164 p.

7. Nielsen M., Introduction to the flowering plant of West Africa, Londres: University of London Press Ltd, 1979, $90 \mathrm{p}$.

8. Irvine F.R., Woody plants of Ghana with special reference to their uses, Londres : Oxford University Press, 1961, 13-14.

9. Babajide O., Olusegun E., Into L., and Raimo H., Constituents of the essential Oil of Monodora tenuifolia (Benth.)W. Asch Root, Flavour and Fragrance Journal, 4, 1989, 193-195.

10. Bouquet A. et Debray M., Plantes médicinales de Côte d'Ivoire, 1974, Éd. O.R.S.T.O.M., Paris (France).

11. Feyisayo A.K. and Oluokun O.O., Comparative analysis of phenolic profile of Monodora myristica and Monodora tenuifolia, African Journal of Agricultural Research, 9, 2014, 1296-1302.

12. Ezenwali M.O., Njoku O.U., and Okoli C.O., Studies on the anti-diarrheal propertis ef seed extract of Monodora tenuifolia, International Journal of Applied Research in Natural Products, 2, 2010, 20-26.

13. Nwaji M.N., Onyiriuka S.O., and Taylor D.A.H., 6-(3-methylbut-1,3-dienyl)indole from Monodora tenuifolia, Journal of the Chemical Society, Chemical Communications, 1972, 327-327.

14. Roques R., Declercq J.P. et Germain G., Acétyl-1-O-Acétyl-6 Laurelliptine, Acta Cryst., B34, 1978, 2017-2020.

15. Lebœuf M., Cavé A., Bhaumik P.K., Mukuherijee B., and Mukherjee R., Phytochemistry, 21, 1982, 2783-2813

16. Fournier G., Leboeuf M., and Cavé A., Annonacea Essential oils : A Review, J. Essent. Oil Res., 11, 1999, 131-142.

17. Ibironké A.A., Vivian N.A., Richard E.A, and Sergio J.M., Evaluation of Monodora tenuifolia seed oil, Annals Food Science and Technology, 13, 2012, 161-167.

18. Adams R.P. Identification of essential oils by ion mass spectroscopy. Academy Press, Inc, New-York. 1989, $302 \mathrm{p}$.

19. Swigar A.A., and Silverstein R.M., Monoterpènes, Infrared, Mass, NMR Spectra and Kovats Indices, Aldrich Chem. Co. Milwaukee, WI, USA, 1981.

20. Affia B.F., Tonzibo Z.F., Koffi A.M., Chalard P. and Figueredo G., Chemical Composition of Essential Oil of Melanthera scandens (Schum. et Thonn), World Applied Sciences Journal, 15, 2011, 992-995.

21. Kesatebrhan H., Tesfahun K., and Aman Dekebo, A comparative study of volatile components of propolis (bee glue) collected from Haramaya university and Assela beekeeping centers, Ethiopia, Bull. Chem. Soc. Ethiop. 26, 2012, 353-360. 
22. Hassane S.O.S., Satrani B., Ghanmi M., Mansouri N., Mohamed H. et Chaouch A., Activité antimicrobienne et composition chimique de l'huile essentielle de Plectranthus aromaticus (Roxb) de l'île de Grande Comore, Biotechno. Agron. Soc. Environ., 15, 2011, 251-258.

23. Veres K., Varga E., Dobos A., Hajdu Zs; Mathe I., Nemeth E., and Szabo K., Investigation of the composition and stability of the essential oil of Origanum, vulgare ssp., vulgare L., and O. vulgare ssp. hirtum (Link) letswaart, Chromatographia, 57, 2003, 95-98. 